\title{
A REMARK ON UNIQUE ERGODICITY AND THE CONTACT TYPE CONDITION
}

\author{
VIKTOR L. GINZBURG AND CÉSAR J. NICHE
}

\begin{abstract}
We prove that for a broad class of exact symplectic manifolds including $\mathbb{R}^{2 m}$ the Hamiltonian flow on a regular compact energy level of an autonomous Hamiltonian cannot be uniquely ergodic. This is a consequence of the Weinstein conjecture and an observation that a Hamiltonian structure with non-vanishing self-linking number must have contact type. We apply these results to show that certain types of exact twisted geodesic flows cannot be uniquely ergodic.
\end{abstract}

\section{Results}

1.1. Introduction. In this paper we show that the Hamiltonian flow on a regular compact energy level of an autonomous Hamiltonian on $\mathbb{R}^{2 m}$ cannot be uniquely ergodic. In fact, the result holds for a much larger class of symplectic manifolds. For instance, it is sufficient to assume that every compact subset of the ambient manifold has finite Hofer-Zehnder capacity.

To put these results in perspective, recall that by the so-called almost existence theorem proved by Hofer and Zehnder, [HZ], and by Struwe, [St], almost all, in the sense of measure theory, regular energy levels of a proper Hamiltonian on $\mathbb{R}^{2 m}$ carry periodic orbits. (Again, this theorem holds for a much broader class of ambient manifolds; see, e.g., [HZ] and [Gi05] for a survey of related results.) On the other hand, it is also known that periodic orbits need not exist on all regular energy levels. To be more specific, when $2 m \geq 4$, there exists a proper Hamiltonian $H: \mathbb{R}^{2 m} \rightarrow \mathbb{R}$ with only one critical point, which is thus a minimum, carrying no periodic orbits of its Hamiltonian flow on a regular level. Hamiltonians with this property are known as counterexamples to the Hamiltonian Seifert conjecture. (The Hamiltonian $H$ is $C^{\infty}$ smooth when $2 m>4$. However, when $2 m=4$, the Hamiltonian is only $C^{2}$ and it is not known if a $C^{\infty}$-smooth $H$ exists.) We refer the reader to the survey [Gi99] and to [GG] for further references and a more detailed discussion of the counterexamples to the Hamiltonian Seifert conjecture.)

This naturally leads to the question of how far from having periodic orbits can the flow on a regular level of $H$ can be. Unique ergodicity is arguably the most extreme form of "aperiodicity", and our results show that the flow on a regular level of a proper Hamiltonian on $\mathbb{R}^{2 m}$ cannot be so "aperiodic". This is also true for many other, but not all, ambient symplectic manifolds; see Corollary 1.3 and Example 1.5.

Date: April 25, 2022.

2010 Mathematics Subject Classification. 53D40, 37J55.

Key words and phrases. Unique ergodicity, Reeb flows, Weinstein conjecture, almost existence theorem, twisted geodesic flows.

The work is partially supported by NSF grant DMS-1308501. 
As an application of this approach, we show that in certain situations exact twisted geodesic flows cannot be uniquely ergodic; see Corollary 1.6.

The proof of these results is rather indirect, although it amounts to a simple combination of well known facts. First, we use the contact type criterion due to McDuff, $[\mathrm{McD}]$, to show that a uniquely ergodic "Hamiltonian structure" meeting a certain natural requirement must have contact type. This is our Theorem 1.1, which is also closely related to a result of Taubes from [Ta09]; see Corollary 1.4. The requirement is that the self-linking number of the structure is non-zero and it is automatically satisfied for a closed hypersurface bounding a domain in an exact symplectic manifold. Next, we observe that whenever the Weinstein conjecture is established for the resulting class of contact type hypersurfaces, the Reeb flow has a closed characteristic, and hence cannot be uniquely ergodic; see Corollaries 1.2 and 1.3. Another way to interpret these results is that certain natural, essentially topological, requirements on a Hamiltonian structure imply a constraint on its dynamics, namely, that the structure cannot be uniquely ergodic.

1.2. Main Results. Before stating the main results of this note, let us introduce some terminology.

Let $M^{2 n+1}$ be a closed oriented manifold and $\omega$ be a maximally non-degenerate (i.e., $\omega^{n} \neq 0$ anywhere on $M$ ) exact two-form on $M$. For brevity, we will refer to $\omega$ as a Hamiltonian structure, cf. [EKP]. (We emphasize that we do not impose any further conditions, e.g., stability, on $\omega$.) An example of a Hamiltonian structure is the restriction of a symplectic form to a compact regular level of a Hamiltonian when the symplectic form on the level is exact. Note that for a fixed volume form $\mu$ on $M$ the non-vanishing vector field $X$ determined by the condition $i_{X} \mu=\omega^{n}$ is necessarily volume preserving. Conversely, in dimension three, once $M$ and $\mu$ are fixed, $X \mapsto i_{X} \mu$ is a one-to-one correspondence between exact divergence-free vector fields and Hamiltonian structures. (In higher dimensions, a non-vanishing divergence-free vector field $X$ such that $i_{X} \mu$ is exact need not come from a Hamiltonian structure.) In any event, a Hamiltonian structure gives rise to the line field ker $\omega$ which integrates to a transversely symplectic one-dimensional foliation $\mathcal{F}$ called the characteristic foliation of $\omega$. The leaves of $\mathcal{F}$ are the integral curves of $X$. Recall that a flow is said to be uniquely ergodic if it admits only one ergodic measure. For volume preserving flows this must then be the volume form. The flow is minimal if its every orbit is dense. (See, e.g., $[\mathrm{KH}]$ for further details.) We call $\omega$ uniquely ergodic (minimal) when $\mathcal{F}$, i.e., the flow of $X$, is uniquely ergodic (minimal). Finally, we say that $\omega$ has contact type when $\omega$ has a contact primitive, i.e., there exists a one-form $\alpha$ such that $d \alpha=\omega$ and $\alpha \wedge(d \alpha)^{n} \neq 0$ everywhere.

To a Hamiltonian structure $\omega$ we associate a number

$$
\operatorname{Lk}(\omega)=\int_{M} \alpha \wedge \omega^{n},
$$

where $\alpha$ is a primitive of $\omega$. Since $M$ is closed, $\operatorname{Lk}(\omega)$ is well defined, i.e., independent of $\alpha$, by the Stokes' formula. (The sign of $\operatorname{Lk}(\omega)$ depends on the orientation of M.) Clearly, $\operatorname{Lk}(\omega) \neq 0$ when $\omega$ has contact type. In dimension three, $\operatorname{Lk}(\omega)$ can be interpreted as the self-linking number of $\mathcal{F}$ or the asymptotic Hopf invariant; see $[$ Ar86].

Our key technical result is the following theorem. 
Theorem 1.1. Let $\omega$ be a uniquely ergodic Hamiltonian structure with $\operatorname{Lk}(\omega) \neq 0$. Then $\omega$ has contact type.

The proof of this theorem, given in detail in Section 2, is an easy application of the contact type criterion from $[\mathrm{McD}]$. The theorem is essentially a negative result saying that a Hamiltonian structure with $\operatorname{Lk}(\omega) \neq 0$ can not be uniquely ergodic if we assume the Weinstein conjecture to hold. Indeed, the Weinstein conjecture asserts that the characteristic foliation of a contact type Hamiltonian structure has a periodic orbit, and hence cannot be uniquely ergodic. (See, e.g., [Hu] for a detailed discussion). Thus this is really so in the cases where the Weinstein conjecture has been established, e.g., when $M$ is three-dimensional, [Ta07], or when $M$ is a hypersurface in $\mathbb{R}^{2 m}$, [Vi], or even more generally $M$ is a displaceable hypersurface in a sufficiently nice symplectic manifold; see, e.g., [Gi05, Hu, HZ] for further references. Moreover, in some instances, the condition that $\operatorname{Lk}(\omega) \neq 0$ is satisfied automatically. For example, we have

Corollary 1.2. Let $M$ be a connected closed hypersurface in $\mathbb{R}^{2 m}$. Then the characteristic foliation on $M$ cannot be uniquely ergodic. Equivalently, an autonomous Hamiltonian flow on a connected regular level of a proper Hamiltonian on $\mathbb{R}^{2 m}$ cannot be uniquely ergodic.

Proof. Let $W$ be the domain bounded by $M$ in $\mathbb{R}^{2 m}$ and let $M=\partial W$ be oriented by the outward normal. Let us also denote by $\omega$ the standard symplectic form on $\mathbb{R}^{2 m}$. The result is then an immediate consequence of Theorem 1.1, the Weinstein conjecture for hypersurfaces in $\mathbb{R}^{2 m}$ mentioned above, and the equality

$$
\operatorname{Lk}(\omega)=\int_{W} \omega^{m}>0,
$$

which in turn readily follows from the Stokes' formula.

This simple observation deserves a further discussion. First, we note for future reference that a much more general result holds.

Corollary 1.3. Let $M$ be a connected closed hypersurface in an exact geometrically bounded symplectic manifold $V$ bounding a domain $W$ with finite Hofer-Zehnder capacity. Then the characteristic foliation on $M$ cannot be uniquely ergodic. In particular, if every compact set in $V$ has finite Hofer-Zehnder capacity (e.g., is displaceable), an autonomous Hamiltonian flow on a connected regular level of a proper, bounded from below Hamiltonian on $V$ cannot be uniquely ergodic.

We refer the reader to, e.g., [Gi05, HZ] for the definitions and notions used in the corollary, noting here only that the conditions on $V$ and $W$ are automatically satisfied when $V$ is a subcritical symplectic Stein manifold. The proof of this corollary is identical to the proof of Corollary 1.2, but now we use the fact that the Weinstein conjecture holds for $M$ since $W$ has finite Hofer-Zehnder capacity; see [HZ]. The corollary can be further generalized; however, this variant is more than sufficient for our purposes.

Next, recall that a uniquely ergodic volume-preserving flow is automatically minimal; see, e.g., $[\mathrm{KH}]$. The converse is not true; see [Fu61] and also $[\mathrm{KH}]$ for further details. (However, the authors are not aware of any example of a uniquely ergodic, but not minimal, Hamiltonian structure $\omega$ with $\operatorname{Lk}(\omega) \neq 0$.) It is tempting to conjecture that in the setting of Theorem 1.1, or at least in the context of Corollaries 
1.2 and 1.3, the Hamiltonian structure cannot be minimal. This would be a much deeper and more difficult result than Theorem 1.1. For hypersurfaces in $\mathbb{R}^{4}$, a proof of this fact was recently announced by Fish and Hofer, [FH].

Turning to another application of Theorem 1.1, we have

Corollary 1.4 (Taubes, [Ta09]). Let $\omega$ be a Hamiltonian structure on a closed (oriented) 3-manifold $M$ with $\operatorname{Lk}(\omega) \neq 0$. Then $\omega$ is not uniquely ergodic.

This corollary is an immediate consequence of Theorem 1.1 and, on the nontrivial side, the Weinstein conjecture for 3-manifolds proved by Taubes in [Ta07]; see also $[\mathrm{Hu}]$ for a survey of related results. Here the new point is that to establish the corollary we use the assertion of the Weinstein conjecture rather than its proof as in [Ta09].

1.3. Applications and Examples: Twisted Geodesic Flows. Let $\sigma$ be a closed 2-form (a magnetic field) on a closed Riemannian manifold $B$. We equip $T^{*} B$ with the twisted symplectic structure $\omega=\omega_{0}+\pi^{*} \sigma$, where $\omega_{0}$ is the standard symplectic form on $T^{*} B$ and $\pi: T^{*} M \rightarrow B$ is the natural projection, and let $K$ be the standard kinetic energy Hamiltonian on $T^{*} B$ arising from the Riemannian metric on $B$. The Hamiltonian flow of $K$ on $T^{*} B$ governs the motion of a charge on $B$ in the magnetic field $\sigma$ and is referred to as a twisted geodesic flow. In contrast with the geodesic flow (the case $\sigma=0$ ), the dynamics of a twisted geodesic flow on an energy level $M_{\epsilon}=\left\{K=\epsilon^{2} / 2\right\}$ depends on the level.

Example 1.5 (Horocycle flow). Let $B$ be a closed surface equipped with a metric of constant negative curvature -1 and let $\sigma$ be the area form on $B$. Note that the restriction $\left.\omega\right|_{M_{\epsilon}}$ is exact for every $\epsilon>0$ although the form $\omega$ is not exact on $T^{*} B$. When $0<\epsilon<1$, every orbit of the Hamiltonian flow on $M_{\epsilon}$ is closed and all orbits have the same period. When $\epsilon>1$, the flow on $M$ is smoothly conjugate to the geodesic flow, up to a time change. The flow on $M_{1}$ is the so-called horocycle flow. (This observation goes back to [Ar61].) The horocycle flow is known to be uniquely ergodic, [Fu73], and, as is easy to see, $\operatorname{Lk}\left(\left.\omega\right|_{M_{1}}\right)=0$, which shows that the conditions that $\operatorname{Lk}(\omega) \neq 0$ in Theorem 1.1 and that $V$ is exact in Corollary 1.3 are essential.

Corollary 1.6. Assume that the form $\sigma$ is exact. Then, for $\epsilon>0$ sufficiently small, the Hamiltonian flow on $M_{\epsilon}$ cannot be uniquely ergodic.

This corollary is an immediate consequence of Corollary 1.3 and a theorem of Schlenk, [Sc], asserting that a small neighborhood of the zero section in $T^{*} B$ has finite Hofer-Zehnder capacity.

Remark 1.7. Corollary 1.6 can also be generalized in a variety of ways. For instance, one can replace the level $M_{\epsilon}$ of $K$ by any closed hypersurface in a sufficiently small neighborhood of the zero section. Furthermore, $T^{*} B$ can be replaced by any exact geometrically bounded symplectic manifold, meeting some minor additional requirements, and the zero section can be replaced by any submanifold $B$ such that $\left.\omega\right|_{B} \neq 0$, cf. [Gü].

\section{Proof of Theorem 1.1}

As has been mentioned in Section 1.2, the theorem readily follows from McDuff's contact type criterion, [McD], based, in turn, on a work of Sullivan, [Su]. To state this criterion, we need first to introduce several notions. 
Fix a volume form $\mu$ on $M$ and let $X$ be the vector field uniquely determined by the condition $i_{X} \mu=\omega^{n}$. The integral curves of the flow of $X$ are parametrized characteristics of $\omega$. Consider the currents of the form $X \otimes \nu$, where $\nu$ is an invariant measure on $M$, acting on a 1 -form $\alpha$ as

$$
\langle X \otimes \nu, \alpha\rangle:=\int_{M} \alpha(X) d \nu,
$$

By definition, such a current is a structure boundary if

$$
\langle X \otimes \nu, \beta\rangle=0
$$

whenever $\beta$ is closed; see $[\mathrm{McD}, \mathrm{Su}]$. For instance, a contractible periodic orbit of $X$, i.e., a closed characteristic of $\omega$, gives rise to a structure boundary which is simply the integral over the orbit.

Let $\alpha$ be a primitive of $\omega$. To a structure boundary we can associate its "action"

$$
A(X \otimes \nu):=\langle X \otimes \nu, \alpha\rangle
$$

which is clearly independent of the choice of $\alpha$. McDuff's contact type criterion asserts that $\omega$ has contact type if and only if

$$
A(X \otimes \nu) \neq 0
$$

for all structure boundaries $X \otimes \nu$; see $[\mathrm{McD}]$. (The observation that the actions on all contractible closed characteristics must have the same sign for a closed contact type hypersurface goes back to [We], where it is used to construct hypersurfaces in $\mathbb{R}^{2 m}$ which do not have contact type.)

In the setting of the theorem, $\mu$ is the only invariant measure since $\omega$ (and hence $X)$ are uniquely ergodic. Thus $X \otimes \mu$ is the only candidate for a structure boundary and, in fact, it is a structure boundary. Indeed, assume that $d \beta=0$. Then, since $i_{X} \mu=\omega^{n}$ and $d \alpha=\omega$, we have

$$
\begin{aligned}
\langle X \otimes \mu, \beta\rangle & =\int_{M} \beta(X) d \mu \\
& =-\int_{M} \beta \wedge(d \alpha)^{n} \\
& =\int_{M} d\left[\beta \wedge \alpha \wedge(d \alpha)^{n-1}\right] \\
& =0
\end{aligned}
$$

by Stokes' formula. (Alternatively, one can argue that $X \otimes \mu$ must be a structure boundary since structure boundaries always exists; see [Su].)

In a similar vein, we have

$$
A(X \otimes \mu)=\int_{M} \alpha(X) d \mu=-\int_{M} \alpha \wedge \omega^{n}=-\operatorname{Lk}(\omega) \neq 0
$$

by the hypotheses of the theorem. Hence, the conditions of McDuff's criterion are met and $(M, \omega)$ has contact type.

Acknowledgements. The authors are grateful to Başak Gürel for useful discussions. A part of this work was carried out while the first author was visiting the ICMAT, Madrid, and he would like to thank the ICMAT for its warm hospitality and support. 


\section{REFERENCES}

[Ar61] V.I. Arnold, Some remarks on flows of line elements and frames, (Russian) Dokl. Akad. Nauk SSSR, 138 (1961), 255-257.

[Ar86] V.I. Arnold, The asymptotic Hopf invariant and its applications, Selecta Math. Soviet., 5 (1986), 327-345.

[EKP] Ya. Eliashberg, S.S. Kim, L. Polterovich, Geometry of contact transformations and domains: orderability versus squeezing, Geom. Topol., 10 (2006), 1635-1747.

$[\mathrm{FH}] \quad$ J. Fish, H. Hofer, work in progress.

[Fu61] H. Furstenberg, Strict ergodicity and transformation of the torus, Amer. J. Math., 83 (1961), 573-601.

[Fu73] H. Furstenberg, The unique ergodicity of the horocycle flow, in Recent advances in topological dynamics (Proc. Conf., Yale Univ., New Haven, Conn., 1972; in honor of Gustav Arnold Hedlund), pp. 95-115. Lecture Notes in Math., Vol. 318, Springer, Berlin, 1973.

[Gi99] V.L. Ginzburg, Hamiltonian dynamical systems without periodic orbits, in Northern California Symplectic Geometry Seminar, 35-48, Amer. Math. Soc. Transl. Ser. 2, 196, Amer. Math. Soc., Providence, RI, 1999.

[Gi05] V.L. Ginzburg, The Weinstein conjecture and theorems of nearby and almost existence, in The Breadth of Symplectic and Poisson Geometry, 139-172, Progr. Math., 232, Birkhäuser Boston, Boston, MA, 2005.

[GG] V.L. Ginzburg, B.Z. Gürel, A $C^{2}$-smooth counterexample to the Hamiltonian Seifert conjecture in $\mathbb{R}^{4}$, Ann. of Math. (2), 158 (2003), 953-976.

[Gü] B.Z. Gürel, Totally non-coisotropic displacement and its applications to Hamiltonian dynamics, Comm. Contemp. Math., 10 (2008), 1103-1128.

[HZ] H. Hofer, E. Zehnder, Symplectic Invariants and Hamiltonian Dynamics, Birkäuser Verlag, Basel, 1994.

$[\mathrm{Hu}]$ M. Hutchings, Taubes's proof of the Weinstein conjecture in dimension three, Bull. Amer. Math. Soc. (N.S.), 47 (2010), 73-125.

[KH] A. Katok, B. Hasselblatt, Introduction to the Modern Theory of Dynamical Systems, Encyc. of Mathematics and its Applications, 54, Cambridge Univ. Press, Cambridge, 1995.

[McD] D. McDuff, Applications of convex integration to symplectic and contact geometry, Ann. Inst. Fourier (Grenoble), 37 (1987), 107-133.

[Sc] F. Schlenk, Applications of Hofers geometry to Hamiltonian dynamics, Comment. Math. Helv., 81 (2006), 105-121.

[St] M. Struwe, Existence of periodic solutions of Hamiltonian systems on almost every energy surfaces, Bol. Soc. Bras. Mat., 20 (1990), 49-58.

[Su] D. Sullivan, Cycles for the dynamical study of foliated manifolds and complex manifolds, Invent. Math., 36 (1976), 225-255.

[Ta07] C.H. Taubes, The Seiberg-Witten equations and the Weinstein conjecture, Geom. Topol., 11 (2007), 2117-2202.

[Ta09] C.H. Taubes, An observation concerning uniquely ergodic vector fields on 3-manifolds, J. Gökova Geom. Topol. GGT, 3 (2009), 9-21.

[Vi] C. Viterbo, A proof of Weinstein's conjecture in $\mathbb{R}^{2 n}$, Ann. Inst. H. Poincaré Anal. Non Linéaire, 4 (1987), 337-356.

[We] A. Weinstein, On the hypotheses of Rabinowitz' periodic orbit theorems, J. Differential Equations, 33 (1979), 353-358.

VG: Department of Mathematics, UC Santa Cruz, Santa Cruz, CA 95064, USA

E-mail address: ginzburg@ucsc.edu

CN: Departamento de Matemática Aplicada, Universidade Federal do Rio de Janeiro, Rio DE JANEIRO, RJ - CEP 21941-909, Brasil

E-mail address: cniche@im.ufrj.br 